\title{
Is self-reported insomnia a risk factor for subclinical carotid atherosclerosis?
}

\author{
Lyudmila Korostovtseva', Asiyat Alieva', Oxana Rotar', Mikhail Bochkarev', \\ Maria Boyarinova', Nadezhda Zvartau, ${ }^{1,3}$, Yurii Sviryaev ${ }^{1,2}$, Aleksandra Konradi ${ }^{1,3}$, \\ Eugene Shlyakhto ${ }^{1}$
}

\author{
${ }^{1}$ Almazov National Medical Research Centre, St. Petersburg, Russia \\ ${ }^{2}$ Sechenov Institute of Evolutionary Physiology and Biochemistry of RAS, St. Petersburg, Russia \\ ${ }^{3}$ ITMO University, St. Petersburg, Russia
}

\begin{abstract}
Background. Insomnia is considered an additional cardiovascular (CV) risk factor (RF). However, the data on its association with vascular damage is controversial. We analyzed the relation between self-reported insomnia and carotid atherosclerosis in the population-based sample.

Material and methods. We selected 985 (males - 38\%; mean age $45.2 \pm 11.6$ years) participants of the St Petersburg population-based sample (the ESSE-RF study) who did not have previously known CV events. All subjects underwent a structured interview (lifestyle, medical history). Insomnia was diagnosed when complaints occurred $\geq 3$ times/week (the questions: "How often did you have difficulties in falling asleep for $>30 \mathrm{~min}$ after going to bed in the last month?", "How often did you have difficulties in falling asleep after midnight awakening in the last month?"). Subclinical atherosclerosis was assessed by carotid intima-media thickness (IMT): normal $\leq 0.9 \mathrm{~mm}$, increased IMT 0.9-13 mm, IMT > $13 \mathrm{~mm}$ indicated an atherosclerotic plaque.

Results. In total, 209 subjects reported at least one insomnia complaint; 79 participants (8\%) reported both sleeponset and sleep-maintenance problems. IMT thickening was found in 142 subjects (14.4\%). IMT values were higher in those with frequent nocturnal awakenings $v$ s. non-insomniacs $(0.75 \pm 0.18$ vs. $0.71 \pm 0.17 \mathrm{~mm}, \mathrm{p}=0.006)$. They also showed greater prevalence of thickened IMT $\left(\chi^{2}=4.6, p=0.026\right)$. Mean IMT weakly correlated with insomnia complaints $(\rho=0.10, \mathrm{p}=0.002)$, but the association was no longer significant after adjustment for age, BP and total cholesterol level.

Conclusion. We demonstrated an association between insomnia complaints and subclinical carotid atherosclerosis, although we assume it to be weak compared to the common CV RF.

Keywords: insomnia; carotid atherosclerosis; subclinical atherosclerosis; cardiovascular risk factors; cardiovascular prevention
\end{abstract}

Arterial Hypertens. 2019, vol. 23, no. 2, pages: 105-113

DOI: 10.5603/AH.a2019.0007

Address for correspondence: Lyudmila Korostovtseva

Almazov National Medical Research Centre, St. Petersburg, Russia, tel: +7 (812) 7023749, \#005726, fax: +7 (812) 7023733

e-mail: korostovtseva_Is@almazovcentre.ru

VM Copyright $@ 2019$ Via Medica, ISSN 2449-6170 


\section{Introduction}

Insomnia is defined as a subjective feeling of either inability to fall asleep or to maintain sleep or the dissatisfaction of sleep quality, or perception of unrefreshed sleep despite the adequate opportunities to sleep $[1,2]$. To make a diagnosis of insomnia the complaints should occur at least three times per week for at least three months and lead to a distress or impairment in daily functioning. Insomnia is a highly prevalent sleep disorder which is reported by about $30 \%$ in general population. Its prevalence is even higher in elderly subjects and specific patient cohorts (e.g. psychiatric population) [3].

Recent cross-sectional and prospective studies suggest an association between insomnia and cardiovascular disease [4]. A meta-analysis pooling data from 13 prospective studies including over 100000 healthy subjects demonstrated a significantly increased risk of fatal and non-fatal cardiovascular events among subjects who at baseline reported insomnia symptoms [4].

However, there is lack of evidence of the relation between insomnia and vascular atherosclerotic involvement. There are only few studies assessing the presence of subclinical atherosclerosis in insomniacs, and their data are highly controversial [5-9].

Therefore, we aimed at the assessment of the relation between self-reported insomnia and asymptomatic atherosclerosis in a population-based cohort from a large-scale epidemiology study of cardiovascular diseases in the Russian Federation

\section{Material and methods}

The recruitment and selection of the patients for the presented analysis was performed from a sample of the multicenter observational study "Epidemiology of the cardiovascular diseases in different regions of the Russian Federation" (ESSE-RF). The ESSE-RF study is a 2-stage study performed in 16 regions of the Russian Federation (Fig. 1). In the present analysis we included data only from the cross-sectional arm performed in St Petersburg. Stratified random sample ( $\mathrm{n}=1600,25-64$ years old) was formed based on the Kish selection method. All participants signed informed consent. The protocol was approved by the local ethical committee.

For the present analysis we excluded patients who had previously known coronary artery disease, stroke and severe cognitive decline. Therefore, altogether the data of 985 subjects were included in the analysis (Fig. 2, Tab. I).

The survey was accomplished between 1 November 2012 and 25 November 2014. All subjects underwent a structured interview which included 12 modules and considered the demographics data, lifestyle, medical history, complaints, and therapy. Detailed data on the methods used in the ESSE-RF study are described elsewhere $[10,11]$.

The survey included several questions for the assessment of sleep disorders:

1. How often did you have difficulties in falling asleep for $\geq 30 \mathrm{~min}$. after going to bed during the last month?

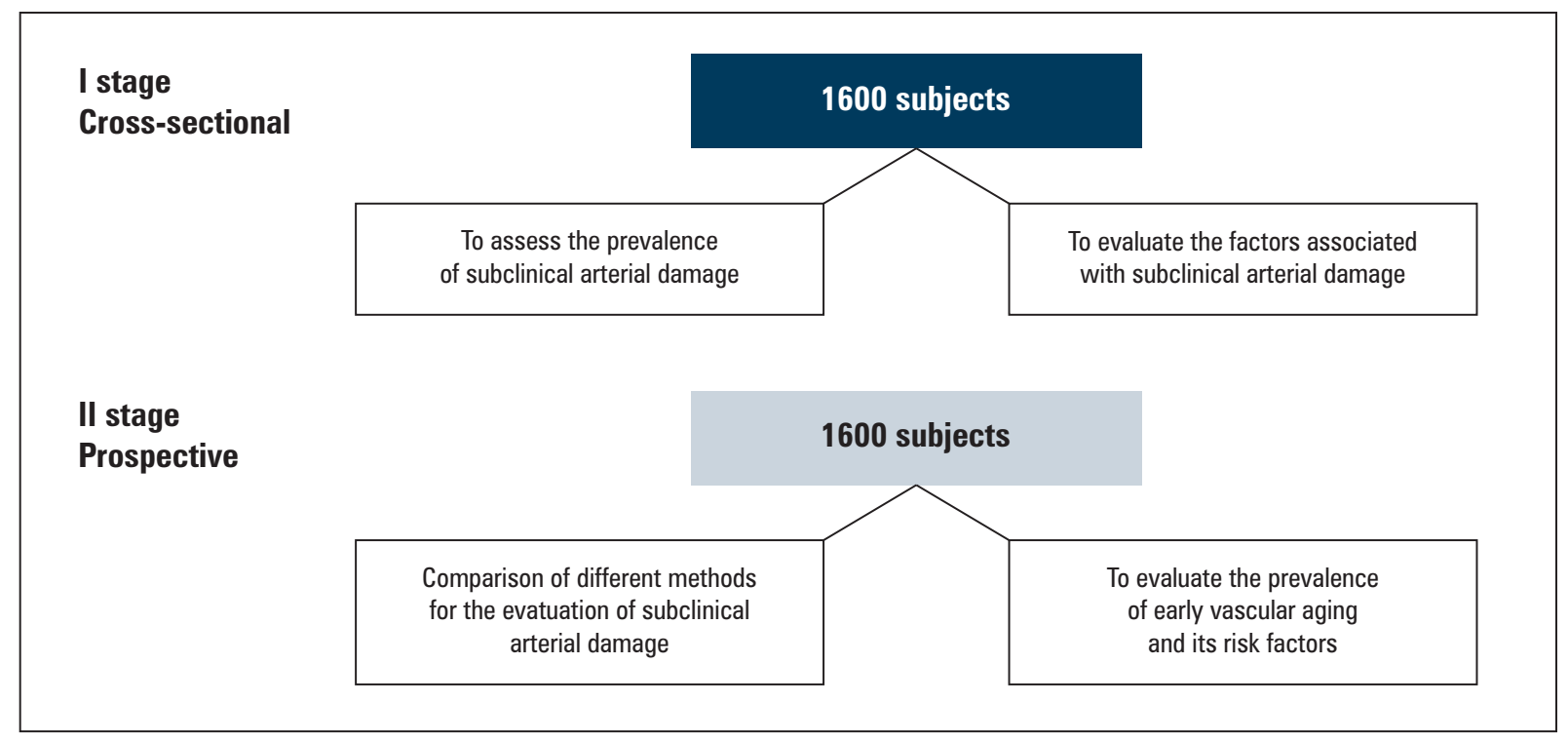

Figure 1. Design of the study „Epidemiology of the cardiovascular diseases in different regions of the Russian Federation” (ESSE-RF study) 


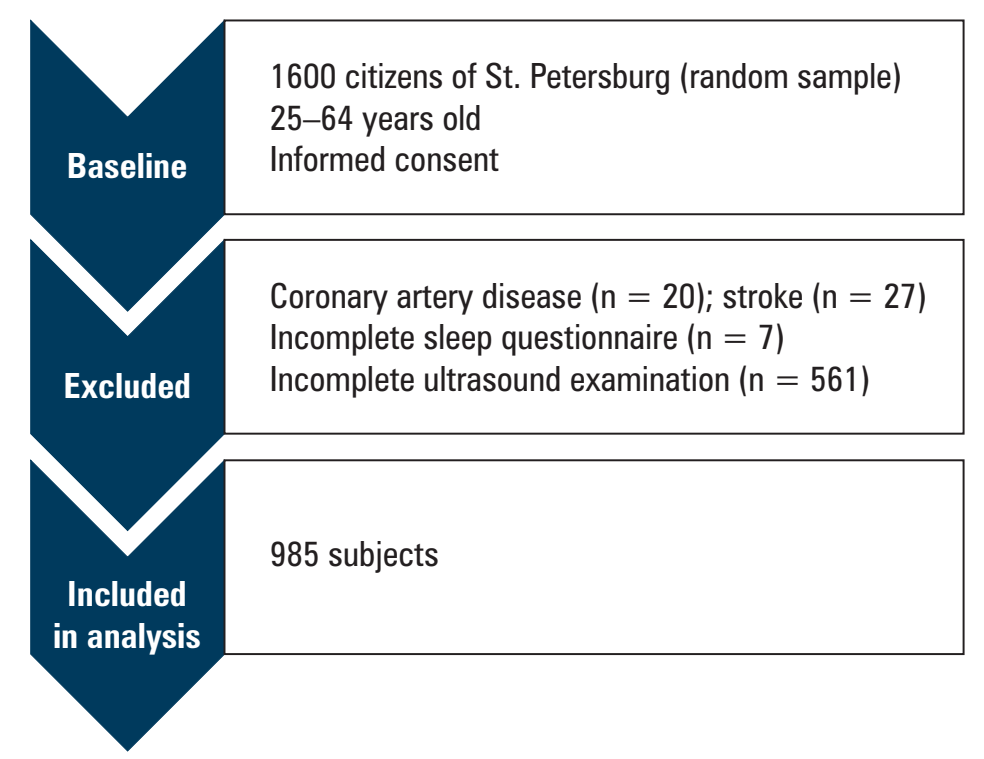

Figure 2. Study sample included in the present analysis

Table I. Clinical and demographic characteristics of the cohort

\begin{tabular}{|l|c|}
\hline Parameter & Value \\
\hline Sex, male/female, $\mathrm{n}(\%)$ & $374(38 \%) / 611(62 \%)$ \\
\hline Age [years] & $45.2 \pm 11.6$ \\
\hline BMI [kg/m²] & $27.1 \pm 5.3$ \\
\hline $\begin{array}{l}\text { Waist circumference } \\
\text { (male/female), [cm] }\end{array}$ & $95.9 \pm 13.2 / 86.4 \pm 14.0$ \\
\hline $\begin{array}{l}\text { Hip circumference } \\
\text { (male/female), [cm] }\end{array}$ & $104.4 \pm 9.2 / 103.6 \pm 10.8$ \\
\hline Obesity (BMI $\left.\geq 30 \mathrm{~kg} / \mathrm{m}^{2}\right), \mathrm{n}(\%)$ & $257(26 \%)$ \\
\hline Hypertension, $\mathrm{n}(\%)$ & $417(42 \%)$ \\
\hline SBP $[\mathrm{mm} \mathrm{Hg}]$ & $127.3 \pm 19.6$ \\
\hline DBP $[\mathrm{mm} \mathrm{Hg]}$ & $78.7 \pm 11.9$ \\
\hline Smoking, $\mathrm{n}(\%)$ & $496(50 \%)$ \\
\hline Total cholesterol [mmol/L] & $5.4 \pm 1.2$ \\
\hline LDL-cholesterol [mmol/L] & $3.5 \pm 1.0$ \\
\hline HDL-cholesterol [mmol/L] & $1.4 \pm 0.4$ \\
\hline Triglycerides [mmol/L] & $1.05(0.2 ; 7.5)$ \\
\hline Dyslipidemia, $\mathrm{n}(\%)$ & $429(44 \%)$ \\
\hline Glucose [mmol/L] & $5.2 \pm 0.9$ \\
\hline Hyperglycemia, $\mathrm{n}(\%)$ & $94(9.5 \%)$ \\
\hline Creatinine [umol/L] & $67.3 \pm 11.8$ \\
\hline eGFR [mL/min/1.73 m²] & $104.0 \pm 12.8$ \\
\hline C-reactive protein [mg/L] & $0.87(0.04 ; 19.87)$ \\
\hline
\end{tabular}

BMI — body mass index; SBP — systolic blood pressure; DBP — diastolic blood pressure; LDL — low density lipoprotein; HDL — high density lipoprotein; eGFR — estimated glomerular filtration rate
2. How often did you have difficulties in falling asleep after midnight awakening during the last month?

3. How often did you have difficulties in staying awake when required?

4. How often did you take sleeping pills during the last month?

The answer options were the following: $1-$ never;

$2-<1$ time per week; $3-1-2$ times per week; $4-\geq 3$ times per week.

Self-reported sleep-disordered breathing were assessed by the following questions "Do you snore?" and "Do you have sleep apnea?" with the answer options "Yes", "No", "Do not know".

Insomnia was evaluated based on the reported sleep-onset problems or nocturnal awakenings which occurred more than 3 times per week during the last month $[1,2]$. The daytime sleepiness was considered when reported more than 3 times per week.

All subjects underwent physical examination which included blood pressure (BP) and heart rate (HR) assessment, anthropometric measurements, and blood tests.

BP was assessed in accordance with the guidelines on the management of arterial hypertension of the European Society of Cardiology and European Society of Hypertension [12-14]. An automatic tonometer "OMRON M3 Expert" (Japan) was used for the BP and HR assessment: two measurements with the 2-minute interval were performed on the right hand after 5 minutes of rest in supine posi- 
tion. Subjects were considered hypertensive in case of systolic BP (SBP) $\geq 140 \mathrm{~mm} \mathrm{Hg}$ and/or diastolic $\mathrm{BP}(\mathrm{DBP}) \geq 90 \mathrm{~mm} \mathrm{Hg}$ or if they received antihypertensive therapy.

Anthropometric measurements included height, weight, waist and hip circumferences. Body mass index (BMI) was calculated by the Quetlet equation: Weight $(\mathrm{kg}) /$ height $^{2}\left(\mathrm{~m}^{2}\right)$. BMI $\geq 30 \mathrm{~kg} / \mathrm{m}^{2}$ was considered obesity.

Blood tests included fasting lipids, creatinine and glucose (Abbott Architect 8000, Roche-diagnostics, USA), C-reactive protein (Cobas Integra 400 plus, Switzerland; Roche-diagnostics). Dyslipidemia was diagnosed when total cholesterol was $5.0 \mathrm{mmol} / \mathrm{L}$ and higher and/or low-density lipoprotein was $3.0 \mathrm{mmol} / \mathrm{L}$ and higher or if the subject took hypolipidemic drugs. Hyperglycemia was diagnosed when fasting glucose exceeded $6.1 \mathrm{mmol} / \mathrm{L}$, diabetes mellitus was diagnosed either from the previous medical history, or if the glucose level was over $7.0 \mathrm{mmol} / \mathrm{L}$.

Glomerular filtration rate was estimated by the CKD-EPI formula [15].

Carotid atherosclerosis was assessed by the trained and experienced specialist using ultrasound technique (MySono U6, Samsung, Korea) according to the same standard protocol and current guidelines. The measurements were performed on both sides at the distance of $1 \mathrm{~cm}$ from the bifurcation of the common carotid artery on posterior wall at three positions (anterior, medial, and posterior longitudinal). Mean intima-media thickness (IMT) was calculated as the mean value of the three measurements on both sides. Carotid arteries were considered normal when intima-media complex was $\leq 0.9 \mathrm{~mm}$.

Atherosclerosis was diagnosed when there was a thickening of intima-media complex more than $0.9 \mathrm{~mm}$, and the focal thickening more than $1.3 \mathrm{~mm}$ indicated atherosclerotic plaque.

For statistical analysis we used parametric statistics. Descriptive statistics included mean and standard deviation values for the normally distributed variables. The data are presented as mean \pm SD. We applied frequency analysis $\left(\chi^{2}\right)$ to assess the contingency between nominal and categorical variables. We used univariate logistic regression analysis to assess the associations between carotid atherosclerosis (as a dichotomous variable) and other parameters. We applied multivariate logistic regression to evaluate the effect of self-reported insomnia on carotid atherosclerosis (IMT as a dichotomous variable). In multivariable models, we adjusted for potential confounders including age, gender, BP, total cholesterol level, low-density lipoprotein (LDL) level, diagnosis of hypertension, diabetes mellitus and hyperlipid- emia, BMI, smoking status. The results are presented as odds ratio $(\mathrm{OR})$ and $95 \%$ confidence interval (95\% CI). The two-sided p-value $<0.05$ was accepted to define the validity of the statistical hypothesis. The software SPSS 20.0 was used to perform statistical analysis.

\section{Results}

\section{Characteristics of the cohort}

In our sample, men comprised $38 \%$ (Tab. I). The mean age of the subjects was $45.2 \pm 11.6$ years. On average the sample was characterized by moderate overweight. The mean BP values were within normal range, but $42 \%$ of the subjects reported known hypertension, and non-controlled SBP $(\geq 140 \mathrm{~mm} \mathrm{Hg})$ or/and DBP ( $\geq 90 \mathrm{~mm} \mathrm{Hg}$ ) was registered in 279 (28\%) subjects. Based on the blood tests, hypercholesterolemia was found in 690 (70\%) subjects. Each $3^{\text {rd }}$ participant had diabetes mellitus, and 94 (9.5\%) subjects had fasting hyperglycemia.

\section{Frequency of the self-reported insomnia symptoms in the cohort}

Based on the survey, every $5^{\text {th }}$ subject reported at least one insomnia complaint; however, both difficulties in falling asleep and midnight awakenings were reported by the minority of the participants (Fig. 3). Sleep-onset problems are more frequent than sleepmaintenance difficulties. Only a small number of the participants reported hypnotic use (less than 3\%) and daytime sleepiness (less than 7\%).

As expected, women were more prevalent in the insomnia group. Also subjects with self-reported insomnia appeared to be older. There were more smokers and hypertensive subjects among insomniacs compared to those without regular insomnia complaints. At the same time, the rates of hypercholesterolemia, hyperglycemia, and obesity were comparable in two groups (Tab. II).

There was no association between having complaints of sleep-onset or sleep-maintenance difficulties and self-reported snoring $\left(\chi^{2}=0.07, \mathrm{p}=0.81\right)$ or sleep apnea $\left(\chi^{2}=1.07, \mathrm{p}=0.38\right)$.

\section{Association between insomnia and carotid atherosclerosis}

Only a minority of the participants $(\mathrm{n}=17,1.7 \%)$ showed atherosclerotic plaques. Nevertheless, atherosclerotic plaques were more frequent among insomniacs, compared to subjects without sleep complaints (Tab. III). Due to the small number of the subjects with plaques, we also analyzed cases of the increased 


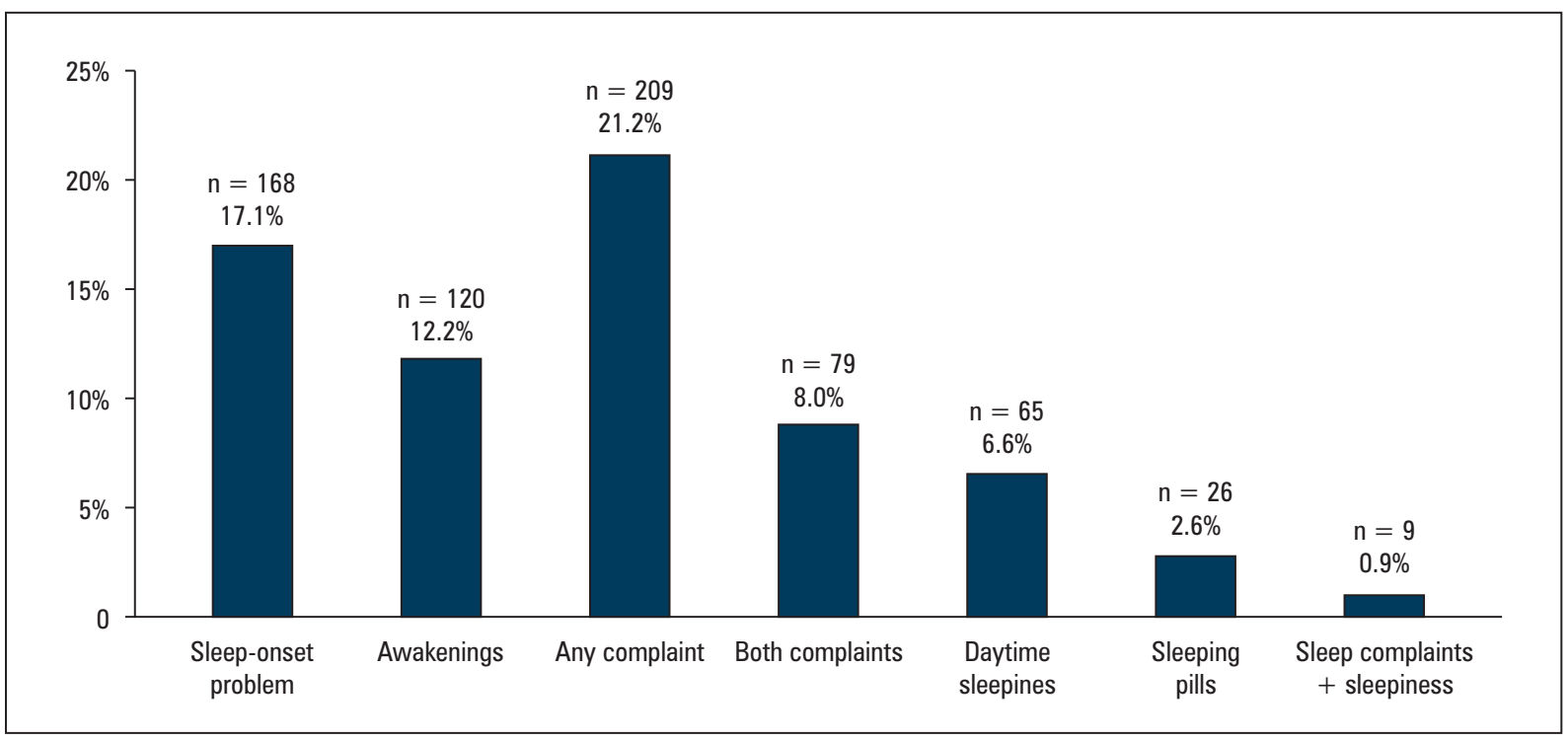

Figure 3. The frequency of self-reported sleep-related complaints in the studied cohort

Table II. Cardiovascular risk factors in insomniacs vs. non-insomniacs

\begin{tabular}{|l|c|c|c|}
\hline Parameter & Insomniacs $(\mathbf{n}=\mathbf{2 0 9})$ & Non-insomniacs $(\mathbf{n}=\mathbf{7 7 6})$ & $\chi^{2}, \mathbf{p}$-level \\
\hline Sex $(\mathrm{m}), \mathrm{n}(\%)$ & $60(29 \%)$ & $642(60 \%)$ & $\chi^{2}=\mathbf{9 . 7} ; \mathbf{p}=\mathbf{0 . 0 0 2}$ \\
\hline Age $($ years) & $48.6 \pm 11.2$ & $44.3 \pm 11.6$ & $\mathbf{p}<\mathbf{0 . 0 0 1}$ \\
\hline BMI $\left[\mathrm{kg} / \mathrm{m}^{2}\right]$ & $27.1 \pm 5.3$ & & $\mathrm{p}=0.46$ \\
\hline $\mathrm{BP}[\mathrm{mm} \mathrm{Hg}]$ & $128 \pm 19 / 78 \pm 11$ & $127 \pm 20 / 79 \pm 12$ & $\mathrm{p}=0.72 / \mathrm{p}=0.73$ \\
\hline Smoking, $\mathrm{n}(\%)$ & $75(36 \%)$ & $305(39 \%)$ & $\chi^{2}=\mathbf{8 . 2} ; \mathbf{p}=\mathbf{0 . 0 1}$ \\
\hline Obesity, $\mathrm{n}(\%)$ & $57(27 \%)$ & $200(26 \%)$ & $\chi^{2}=0.17 ; \mathrm{p}=0.72$ \\
\hline Hypertension, $\mathrm{n}(\%)$ & $115(55 \%)$ & $302(39 \%)$ & $\chi^{2}=\mathbf{1 7 . 4} ; \mathbf{p}<\mathbf{0 . 0 0 1}$ \\
\hline Dyslipidemia, $\mathrm{n}(\%)$ & $147(70 \%)$ & $493(64 \%)$ & $\chi^{2}=3.04 ; \mathrm{p}=0.09$ \\
\hline Hyperglycemia, $\mathrm{n}(\%)$ & $17(8 \%)$ & $77(10 \%)$ & $\chi^{2}=0.64 ; \mathrm{p}=0.43$ \\
\hline
\end{tabular}

$\mathrm{BMI}$ - body mass index; $\mathrm{BP}$ - blood pressure

Table III. The frequency of carotid atherosclerotic lesions in insomniacs vs. non-insomniacs

\begin{tabular}{|l|c|c|c|}
\hline Parameter & Insomniacs $(\mathbf{n}=\mathbf{2 0 9})$ & Non-insomniacs $(\mathbf{n}=\mathbf{7 7 6})$ & $\chi^{2}, \mathbf{p}$-level \\
\hline Atherosclerotic plaques & $7(3.3 \%)$ & $10(1.3 \%)$ & $\chi^{2}=4.12 ; p=0.042$ \\
\hline $\begin{array}{l}\text { Increased intima-media thickness } \\
(>0.9 \mathrm{~mm})\end{array}$ & $39(18.7 \%)$ & $103(13.3 \%)$ & $\chi^{2}=3.87 ; p=0.049$ \\
\hline Total, $\mathrm{n}$ & 209 & 776 & \\
\hline
\end{tabular}

IMT without plaque formation. The rate of abnormal IMT thickening was also higher in insomniacs (Fig. 4).

Increased IMT was more prevalent among those with nocturnal awakenings, while the rate of subclinical atherosclerosis was similar among subjects with and without sleep-onset problems (Fig. 5).

Similarly, IMT values were greater in those with frequent nocturnal awakenings compared to non-in- somniacs $(0.75 \pm 0.18$ vs. $0.71 \pm 0.17 \mathrm{~mm}, \mathrm{p}=0.006)$, although average IMT was within reference range.

Univariate regression analysis demonstrated a weak association between mean IMT and insomnia complaints $(\rho=0.10, \mathrm{p}=0.002)$, and the relation is more significant in subjects with nocturnal awakenings than in participants with sleep-onset problems (Tab. IV). However, the association was no longer 


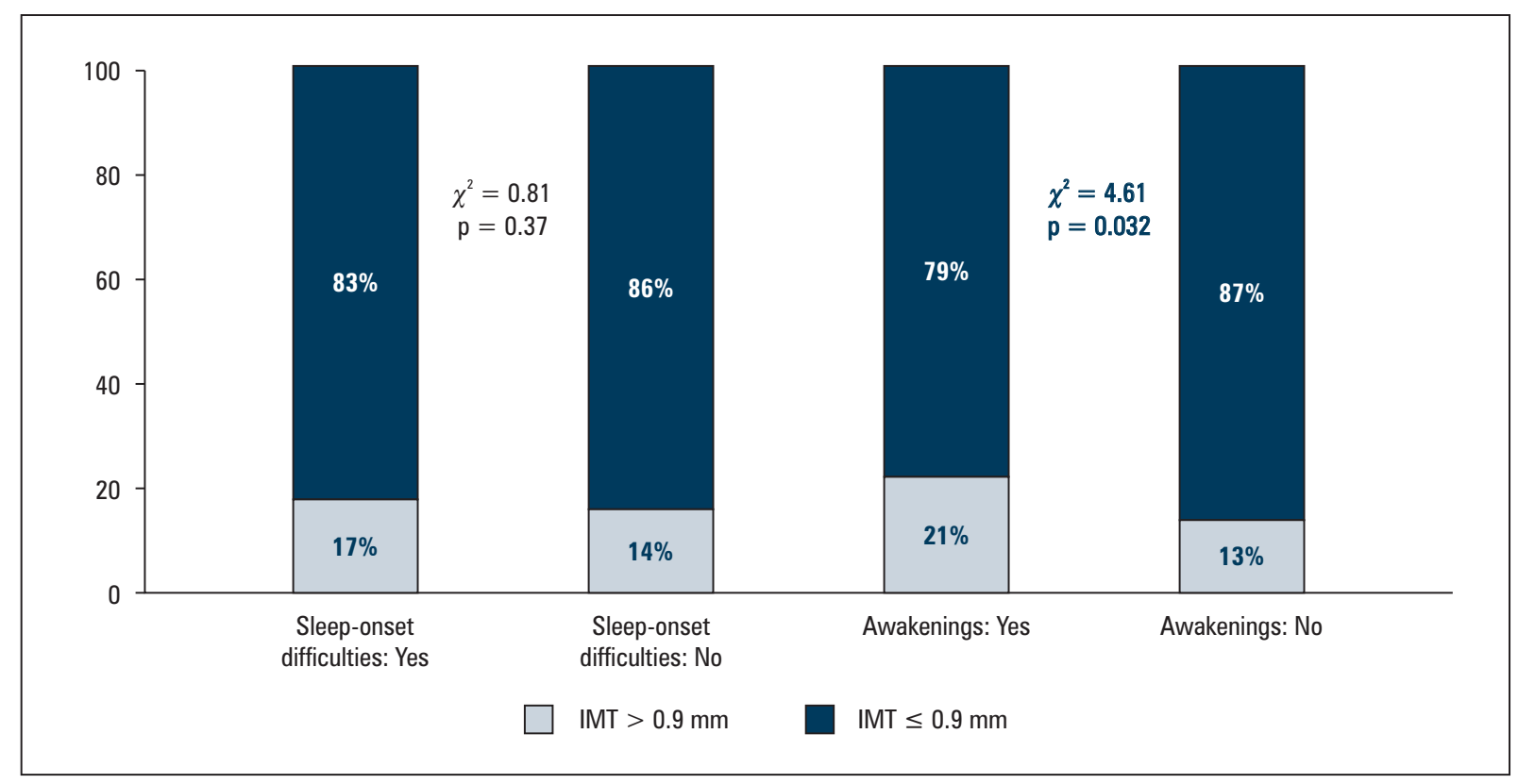

Figure 4. The prevalence of carotid atherosclerosis and self-related insomnia complaints; IMT — intima-media thickness

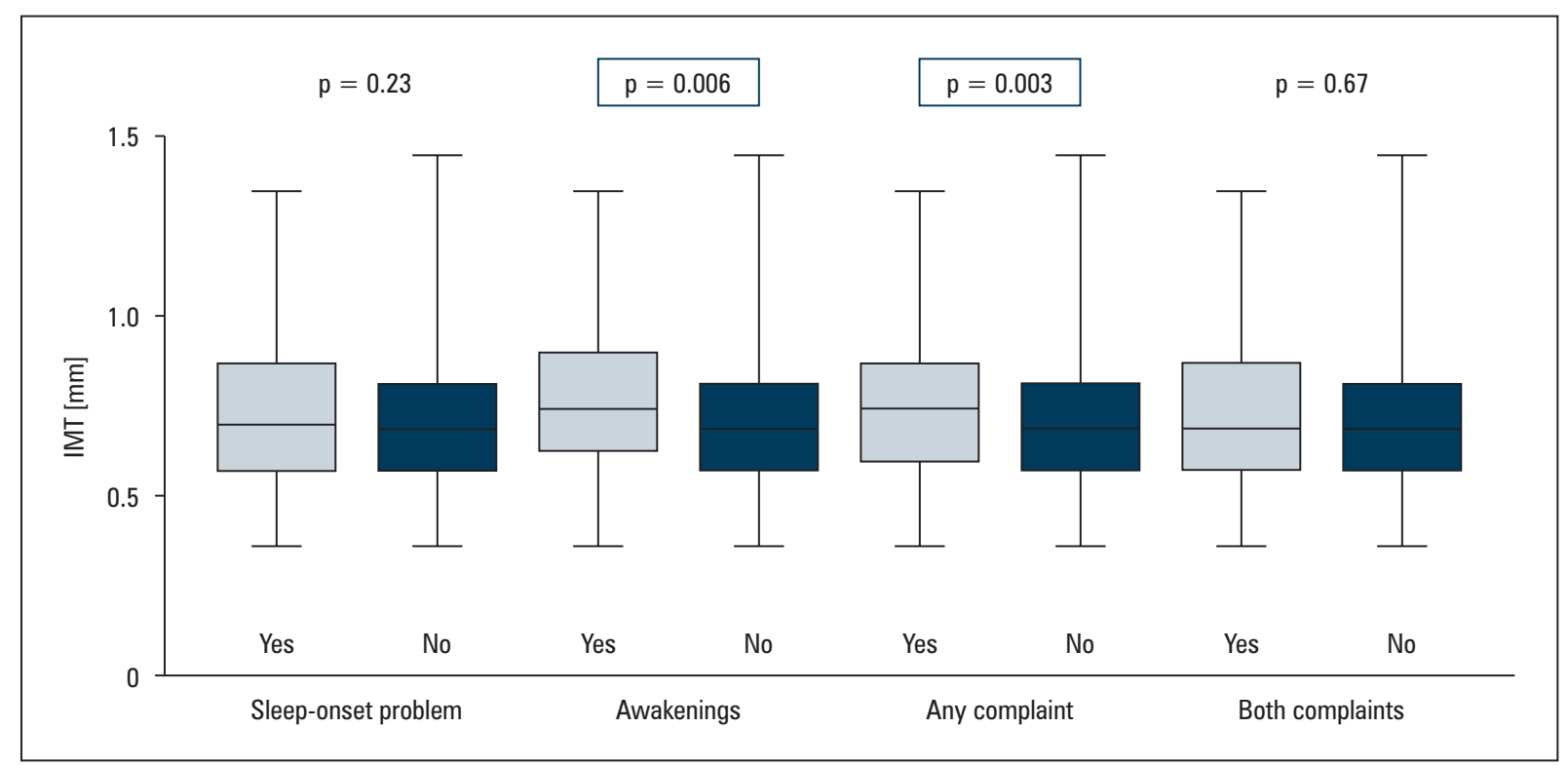

Figure 5. Intima-media thickness (IMT) and insomnia complaints

significant after adjustment for age, BP and total cholesterol level (Tab. V). The adjustment for the hypnotic use did not affect the results.

\section{Discussion}

In our study, in a large adult population cohort without evident cardiovascular disease we have identified a weak univariate association between asymptomatic carotid atherosclerosis and self-reported insomnia. Intriguingly, the nocturnal awakenings show stronger relation to the IMT values than sleep-onset difficulties. However, the role of the common established cardiovascular risk factors (age, BP, cholesterol level) appears to be more significant.

Among few studies which assessed subclinical atherosclerosis in insomnia patients, Ramos-Sepulveda et al. (2010) also did not find an association between self-reported sleep complaints and carotid 
Table IV. Association between self-reported insomnia and carotid atherosclerosis: univariate regression analysis

\begin{tabular}{|l|c|c|}
\hline Insomnia complaints & $\begin{array}{l}\text { IMT mean } \\
\left(\mathbf{R}^{2}, \mathbf{p} \text {-level) }\right.\end{array}$ & OR (95\% CI) \\
\hline $\begin{array}{l}\text { Sleep complaints } \\
\text { (either sleep-onset or sleep-maintenance) }\end{array}$ & $\begin{array}{l}\mathbf{R}^{2}=\mathbf{0 . 0 0 9} \\
\mathbf{p}=\mathbf{0 . 0 0 3}\end{array}$ & $\mathbf{0 . 0 4}(\mathbf{0 . 0 1 4 - 0 . 0 6 5 )}$ \\
\hline $\begin{array}{l}\text { Sleep complaints } \\
\text { (both sleep-onset or sleep-maintenance difficulties) }\end{array}$ & $\mathrm{p}=0.70$ & $0.01(-0.30-0.05)$ \\
\hline Sleep-onset difficulties & $\mathrm{p}=0.27$ & $0.02(-0.01-0.05)$ \\
\hline Awakenings & $\begin{array}{l}\mathbf{R}^{2}=\mathbf{0 . 0 0 8} \\
\mathbf{p}=\mathbf{0 . 0 0 6}\end{array}$ & $\mathbf{0 . 0 5}(\mathbf{0 . 0 1 3 - 0 . 0 7 7 )}$ \\
\hline Sleep complaints + daytime sleepiness & $\begin{array}{l}\mathbf{R}^{2}=\mathbf{0 . 0 0 7} \\
\mathbf{p}=\mathbf{0 . 0 1 1}\end{array}$ & $\mathbf{0 . 1 4}(\mathbf{0 . 0 3 2 - 0 . 2 5 4 )}$ \\
\hline
\end{tabular}

IMT — intima-media thickness; $\mathrm{OR}$ - odds ratio; $\mathrm{Cl}$ - confidence interval

Table V. Association between self-reported insomnia and carotid atherosclerosis: multivariate regression analysis

\begin{tabular}{|l|c|c|c|}
\hline & $\begin{array}{c}\text { Adjusted for age } \\
\text { OR (95\% Cl), p-level }\end{array}$ & $\begin{array}{c}\text { Adjusted for age, SBP } \\
\text { OR (95\% CI), p-level }\end{array}$ & $\begin{array}{c}\text { Adjusted for age, SBP, } \\
\text { LDL-cholesterol } \\
\text { OR (95\% CI), } \mathbf{p} \text {-level }\end{array}$ \\
\hline $\begin{array}{l}\text { Sleep complaints (either sleep-onset } \\
\text { or sleep-maintenance) }\end{array}$ & $\begin{array}{c}\mathrm{p}=0.14 \\
0.006(0.005-0.007)\end{array}$ & $\begin{array}{c}\mathrm{p}=0.12 \\
0.08(-0.02-0.18)\end{array}$ & $\begin{array}{c}\mathrm{p}=0.13 \\
(-0.02-0.18)\end{array}$ \\
\hline Awakenings & $\begin{array}{c}\mathrm{p}=0.52 \\
\mathrm{p}=0.45\end{array}$ & $\begin{array}{c}\mathrm{p}=0.47 \\
0.01(-0.02-0.04)\end{array}$ \\
\hline $\begin{array}{l}\text { Sleep complaints } \\
\text { + daytime sleepiness }\end{array}$ & $\begin{array}{c}\mathrm{p}(-0.02-0.04) \\
\mathrm{p}=0.14\end{array}$ & $\begin{array}{c}\mathrm{p}=0.09 \\
(-0.02-0.04)\end{array}$ & 0.13 \\
\hline
\end{tabular}

$\mathrm{OR}$ — odds ratio; $\mathrm{Cl}$ - confidence interval; $\mathrm{SBP}$ — systolic blood pressure; $\mathrm{LDL}$ - low density lipoprotein

IMT among 1605 middle-aged/elderly (> 40 years old) subjects from the Northern Manhattan Study (NOMAS) cohort [9]. In menopausal women insomnia among other menopausal symptoms was shown to have no association either with carotid IMT or with coronary artery calcification [8].

Potential discrepancy between subjective (selfreported) complaints and true sleep quality might be one of the reasons for the lack of association in our study [16]. We can assume that in some situations the role of insomnia can be greater, e.g. in case of insomnia associated with short sleep duration. Thus, a large population study $(\mathrm{n}=3789)$ by Chen et al. (2017) demonstrated that subjects with short sleep duration (defined as less than 5 hours per night) have higher risk of having carotid plaques when adjusted for other risk factors $(\mathrm{OR}=1.28,95 \% \mathrm{CI}$ : $1.05-1.56, \mathrm{p}<0.001)$ even after stratification for age [17]. The participants over the age of 60 years who reported short sleep duration have the highest risk of carotid plaque (OR $=3.46,95 \% \mathrm{CI}: 2.34-5.12$, $\mathrm{p}<0.01$ ). The study by Nakazaki et al. (2012) also demonstrated that IMT is greater in insomnia subjects; it correlates with SBP, DBP, and total sleep time [5]. Nagai et al. (2013) also found a correlation between persistent insomnia and IMT, and carotid artery stiffness [6]. However, in the latter study only elderly ( $>65$ years, $n=86$ and $>70$ years, $n=201$ ) subjects were included. In our cohort, only 22 subjects reported short sleep duration $(<6$ hours per night) and only six of them demonstrated increased IMT, so due to the small number of cases the further analysis was not performed in this subgroup.

Increased sympathetic activity, higher activity of renin-angiotensin-aldosterone system and hypothalamo-pituitary axis, circadian disorders, impaired insulin sensitivity and others are discussed as potential mechanisms of the association between insomnia and atherosclerosis. These mechanisms can exacerbate hypertension [18], BP variability [6], dyslipidemia contributing to the development and progression of atherosclerosis. We suppose that these effects might be more evident in subjects with more frequent arousals and nocturnal awakenings and can explain our finding of stronger relation to the IMT $[19,20]$.

On the other hand, the lack of association between insomnia and carotid atherosclerosis in our cohort can be also explained by the fact that ultrasound technique provides only a rough evaluation of arterial atherosclerotic involvement when arterial lesion is already advanced, while the negative effects of insomnia can act at the earlier stages. 
Carotid atherosclerosis, in particular, atherosclerotic plaques in carotid arteries, is a well-known cardiovascular risk factor, and according to the recently published 2018 ESC/ESH guidelines for the management of arterial hypertension, carotid plaques are considered a significant modifier of the cardiovascular risk [13]. On the other hand, the European Guidelines on cardiovascular prevention 2016 do not recommend the routine use of imaging techniques for the assessment of increased IMT for risk stratification [21]. At the same time, the guidelines mention, that low predictive value on population level might be related to the lack of standardization in IMT assessment, its high variability and low reproducibility [21]. However, there is a correlation between carotid atherosclerosis and atherosclerotic involvement of other arterial territories, and carotid atherosclerosis remains one of the common tools for the evaluation of arterial disease [22]. Therefore, the use of ultrasound carotid atherosclerosis is reasonable for the assessment of atherosclerosis. In our study, ultrasound study was performed according to the same protocol in all subjects which minimizes the potential variability and bias.

\section{Study limitations}

We recognize that our study has certain limitations. First, our analysis was based on a cross-sectional study, which does not allow making conclusions about causal effects between insomnia and subclinical atherosclerosis. Another important limitation is the lack of objective sleep evaluation which could help to verify and differentiate sleep disorders, as well as to exclude potential discrepancy between subjective and objective sleep parameters [16]. However, insomnia is a diagnosis based on the subjective symptoms [1, 2], and objective methods play only an additional role. We also miss the results about early awakenings due to the limited number of questions in the epidemiological survey. There is a gender imbalance in our insomniacs and non-insomniacs group with the significantly prevalent female population among those reporting insomnia symptoms. However, it corresponds to other studies data which showed that women are more likely to have lower sleep quality and shorter sleep [7, 23].

\section{Conclusion}

In general population, insomniacs demonstrate higher rate of subclinical carotid atherosclerosis includ- ing the frequency of carotid atherosclerotic plaques. Self-reported nocturnal awakenings are stronger associated with the subclinical carotid atherosclerosis compared to the sleep-onset difficulties. In conclusion, our study confirmed an association between insomnia complaints and subclinical carotid atherosclerosis, although this relation appears to be weak compared to the established cardiovascular risk factors, however, deserves further investigation.

\section{Conflict of interest}

The authors declare that there is no conflict of interest regarding the publication of this paper.

\section{Funding statement}

The study was supported by the governmental state program, the analysis and publication were supported by the grant of the Russian Scientific Foundation, project \# 17-75-10099.

\section{Acknowledgements}

The results have been first reported as an oral presentation at the Congress of the International Society of Hypertension 2018 (20-23 September 2018) in Beijing (China); the report was awarded the Austin Doyle Award 2018, the abstract was published in the Conference Proceedings (J Hypertens Vol 36, Suppl.: e223-e224.).

\section{References}

5. International Classification of Sleep Disorders - Third Edition (ICSD-3). American Academy of Sleep Medicine, Darien, USA 2014.

6. Diagnostic and Statistical Manual of Mental Disorders - Fifth Edition (DSM-5TM). American Psychiatric Publishing, Arlington, VA 2013.

7. Seow LS, Verma SK, Mok YM, et al. Evaluating DSM-5 Insomnia Disorder and the Treatment of Sleep Problems in a Psychiatric Population. J Clin Sleep Med. 2018; 14(2): 237-244, doi: 10.5664/ jcsm.6942, indexed in Pubmed: 29394962.

8. Sofi F, Cesari F, Casini A. Insomnia and risk of cardiovascular disease: A meta-analysis. Eur J Prev Cardiol. 2014; 21(1): 57-64, doi: 10.1177/2047487312460020, indexed in Pubmed: 22942213

9. Nakazaki C, Noda A, Koike Y, et al. Association of insomnia and short sleep duration with atherosclerosis risk in the elderly. Am J Hypertens. 2012; 25(11): 1149-1155, doi: 10.1038/ajh.2012.107, indexed in Pubmed: 22854638.

10. Nagai M, Hoshide S, Nishikawa M, et al. Sleep duration and insomnia in the elderly: associations with blood pressure variability and carotid artery remodeling. Am J Hypertens. 2013; 26(8): 981-989, doi: 10.1093/ajh/hpt070, indexed in Pubmed: 23723262.

11. Li CH, Huang KY, Chen WC, et al. Sleep disorders in individuals without sleep apnea increase the risk of peripheral arterial disorder: a nationwide population-based retrospective cohort study. Sleep Med. 2015; 16(8): 966-970, doi: 10.1016/j.sleep.2015.02.538, indexed in Pubmed: 26143166.

12. Wolff EF, He Y, Black DM, et al. Self-reported menopausal symptoms, coronary artery calcification, and carotid intima-media thickness in recently menopausal women screened for the Kronos early estrogen prevention study (KEEPS). Fertil Steril. 2013; 99(5): 
1385-1391, doi: 10.1016/j.fertnstert.2012.11.053, indexed in Pubmed: 23312232.

13. Ramos-Sepulveda A, Wohlgemuth W, Gardener H, et al. Snoring and insomnia are not associated with subclinical atherosclerosis in the Northern Manhattan Study. Int J Stroke. 2010; 5(4): 264-268, doi: 10.1111/j.1747-4949.2010.00438.x, indexed in Pubmed: 20636708.

14. Rotar O, Boyarinova M, Orlov A, et al. Metabolically healthy obese and metabolically unhealthy non-obese phenotypes in a Russian population. Eur J Epidemiol. 2017; 32(3): 251-254, doi: $10.1007 /$ s10654-016-0221-z, indexed in Pubmed: 28039558.

15. Bochkarev MV, Korostovtseva LS, Filchenko IA, et al. [Socialdemographic aspects of insomnia in the Russian population according to ESSE-RF study]. Zh Nevrol Psikhiatr Im S S Korsakova. 2018; 118(4. Vyp. 2): 26-34, doi: 10.17116/jnevro20181184226, indexed in Pubmed: 30059049.

16. Mansia G, De Backer G, Dominiczak A, et al. European Society of Hypertension, European Society of Cardiology, Management of Arterial Hypertension of the European Society of Hypertension, European Society of Cardiology. 2007 Guidelines for the management of arterial hypertension: The Task Force for the Management of Arterial Hypertension of the European Society of Hypertension (ESH) and of the European Society of Cardiology (ESC). Eur Heart J. 2007; 28(12): 1462-1536, doi: 10.1093/eurheartj/ehm236, indexed in Pubmed: 17562668.

17. Williams B, Mancia G, Spiering W, et al. 2018 ESC/ESH Guidelines for the management of arterial hypertension. Eur Heart J. 2018; 39(33): 3021-3104, doi: 10.1093/eurheartj/ehy339, indexed in Pubmed: 30165516.

18. Mancia G, Fagard R, Narkiewicz K, et al. 2013 ESH/ESC guidelines for the management of arterial hypertension: the Task Force for the Management of Arterial Hypertension of the European Society of Hypertension (ESH) and of the European Society of Cardiology (ESC). Eur Heart J. 2013; 34(28): 2159-2219, doi: 10.1093/ eurheartj/eht151, indexed in Pubmed: 23771844.

19. Levey AS, Bosch JP, Lewis JB, et al. A more accurate method to estimate glomerular filtration rate from serum creatinine: a new prediction equation. Modification of Diet in Renal Disease Study Group. Ann Intern Med. 1999; 130(6): 461-470, indexed in Pubmed: 10075613.

20. Kay DB, Buysse DJ, Germain A, et al. Subjective-objective sleep discrepancy among older adults: associations with insomnia diagnosis and insomnia treatment. J Sleep Res. 2015; 24(1): 32-39, doi: $10.1111 /$ jsr.12220, indexed in Pubmed: 25219802.

21. Chen Si, Yang Y, Cheng GL, et al. Association between short sleep duration and carotid atherosclerosis modified by age in a Chinese community population. J Epidemiol Community Health. 2018; 72(6): 539-544, doi: 10.1136/jech-2017-209464, indexed in Pubmed: 29449352.

22. Jarrin DC, Alvaro PK, Bouchard MA, et al. Insomnia and hypertension: A systematic review. Sleep Med Rev. 2018; 41: 3-38, doi: 10.1016/j.smrv.2018.02.003, indexed in Pubmed: 29576408.

23. Słomko J, Zawadka-Kunikowska M, Klawe JJ, et al. Cardiovascular regulation and body temperature: evidence from a nap vs. sleep deprivation randomized controlled trial. Physiol Res. 2018; 67: 687-693, indexed in Pubmed: 30204457.

24. Chouchou F, Pichot V, Pépin JL, et al. PROOF Study Group. Sympathetic overactivity due to sleep fragmentation is associated with elevated diurnal systolic blood pressure in healthy elderly subjects: the PROOF-SYNAPSE study. Eur Heart J. 2013; 34(28): 2122-31, 2131a, doi: 10.1093/eurheartj/eht208, indexed in Pubmed: 23756334

25. Piepoli MF, Hoes AW, Brotons C, et al. Task Force for the 2016 guidelines on cardiovascular disease prevention in clinical practice, en nombre del Comité Español Interdisciplinario para la Prevención Cardiovascular (CEIPC), en nombre del Comité Español Interdisciplinario para la Prevención Cardiovascular (CEIPC), en nombre del Comité Español Interdisciplinario para la Prevención Cardiovascular (CEIPC), en nombre del Comité Español Interdisciplinario para la Prevención Cardiovascular (CEIPC), Comité Español Interdisciplinario para la Prevencón Cardiovascular CEIPC, Authors/Task Force Members:, Authors/Task Force Members, Additional Contributor: Simone Binno (Italy), Document Reviewers:, ESC Scientific Document Group. 2016 European Guidelines on cardiovascular disease prevention in clinical practice: The Sixth Joint Task Force of the European Society of Cardiology and Other Societies on Cardiovascular Disease Prevention in Clinical Practice (constituted by representatives of 10 societies and by invited experts) Developed with the special contribution of the European Association for Cardiovascular Prevention \& Rehabilitation (EACPR). Eur Heart J. 2016; 37(29): 2315-2381, doi: 10.1093/eurheartj/ ehw106, indexed in Pubmed: 27222591.

26. O'Leary DH, Polak JF, Kronmal RA, et al. Carotid-artery intima and media thickness as a risk factor for myocardial infarction and stroke in older adults. Cardiovascular Health Study Collaborative Research Group. N Engl J Med. 1999; 340(1): 14-22, doi: 10.1056/ NEJM199901073400103, indexed in Pubmed: 9878640.

27. van der Berg JF, Miedema H, Tulen J, et al. Sex Differences in Subjective and Actigraphic Sleep Measures: A Population-Based Study of Elderly Persons. Sleep. 2009; 32(10): 1367-1375, doi: 10.1093/ sleep/32.10.1367, indexed in Pubmed: 19848365. 